\title{
Chitosan/iron oxide nanocomposite films: Effect of the composition and preparation methods on the adsorption of congo red
}

\author{
Gianina A. Kloster, Mirna A. Mosiewicki , Norma E. Marcovich * \\ Instituto de Investigaciones en Ciencia y Tecnología de Materiales (INTEMA), Facultad de Ingeniería, Universidad Nacional de Mar del Plata - CONICET, Mar del Plata, Argentina
}

\section{ART ICLE INFO}

\section{Keywords:}

Chitosan

Magnetic chitosan fims

Biosorbent

Congo red

\begin{abstract}
A B S T R A C T
Composite films based on chitosan, glycerol and magnetic iron oxides were obtained by two different procedures: in situ generation of iron oxide particles in the already formed films and dispersion of previously synthesized iron oxide particles into the film forming solution by sonication, and then tested as adsorbents for the removal of congo red (CR) from solutions of different concentrations. Their performances were compared with those of the corresponding films without particles. All the samples presented similar behavior when contacted with solutions containing up to $70 \mathrm{mg} / \mathrm{L}$ CR (maximum sorption capacity $\sim 25 \mathrm{mg} / \mathrm{g}$ ), but sonicated samples exhibited an increased dye adsorption when in contact with more concentrated solutions, reaching up to $700 \mathrm{mg} / \mathrm{g}$ sorption capacity. These differences were explained considering the obtaining procedure of the films, their composition and microstructure, as well as the changes in the $\mathrm{pH}$ of the dye solution once the adsorbent film was put inside.
\end{abstract}

\section{Introduction}

Organic dyes are widely used in many industries due to their bright color, excellent colorfastness and ease of application. The worldwide annual production of dyes is about 700 thousand tons, and about $5-15 \%$ of the dyes are discharged into the waste streams by the textile industries alone (Yang et al., 2018). Due to their toxic, carcinogenic and mutagenic effects on aquatic species and human beings, the presence of dyes in water is a serious environmental problem (Yang et al., 2018). Thus, dyeing wastewater has drawn great public concerns not only owing to its strong coloration, but also due to its high toxicity, carcinogenicity, degradation resistance and easy accumulation in living organisms (Hui et al., 2018; Yang et al., 2018; You et al., 2018). Wastewaters containing dyes are very difficult to treat, since the dyes are recalcitrant molecules, resistant to aerobic digestion. Another difficulty is treatment of wastewaters containing low concentrations of dye molecules, even when the presence of very small amounts of dyes in water is highly visible and undesirable (Zhu, Zhang, Liu, Zhang, \& Han, 2012). Hence, the treatment of polluted, dye-containing water using economically feasible and eco-friendly techniques before being released into the environment is necessary (Thakur, Pandey, \& Arotiba, 2017). Various methods based on membrane technologies, adsorption, biodegradation, distillation, photocatalysis and other approaches, have

* Corresponding author.

Email address: marcovic@fi.mdp.edu.ar (N.E. Marcovich) been utilized for purification and remediation of the polluted water (Hui et al., 2018; You et al., 2018). Among these approaches, adsorption process is regarded as an efficient alternative, due to its simplicity of design, wide adaptability, convenience and ease of operation, especially when the adsorbent is relatively low-priced and readily available (Li et al., 2017; Rafatullah et al., 2010; Thakur et al., 2017; Zahir et al., 2017; Zhou et al., 2018). However, developing the adsorbents with low cost, easy preparation and high efficiency is still the problem and challenge in dye adsorption application (Yang et al., 2018). Recently, it has been also demonstrated that the adsorption of dyes by means of natural and biodegradable polymers is one of the emerging methods for dye removal (Yang et al., 2018). In particular, polysaccharides-based adsorbents are being utilized for the treatment of wastewater from dye industry (Li et al., 2017; Thakur et al., 2017) due to their easy availability and outstanding removal capacities for wide range of dyes (Thakur, Pandey, \& Arotiba, 2016; Zahir et al., 2017). Moreover, to improve the extraction properties of the polysaccharides based adsorbents, nanocomposites-based on these polymers have been used extensively as adsorbents in order to produce a synergistic effect of an inorganic filler and the polysaccharide matrix (Thakur et al., 2017).

Chitosan is a polysaccharide of glucosamine and $\mathrm{N}$-acetyl-D-glucosamine linked together by $\beta(1 \rightarrow 4)$ glycosidic bonds produced by the deacetylation of chitin (Galhoum et al., 2015; Yang et al., 2018; Zahir et al., 2017), which is one of the most abundant biopolymers. This nat- 


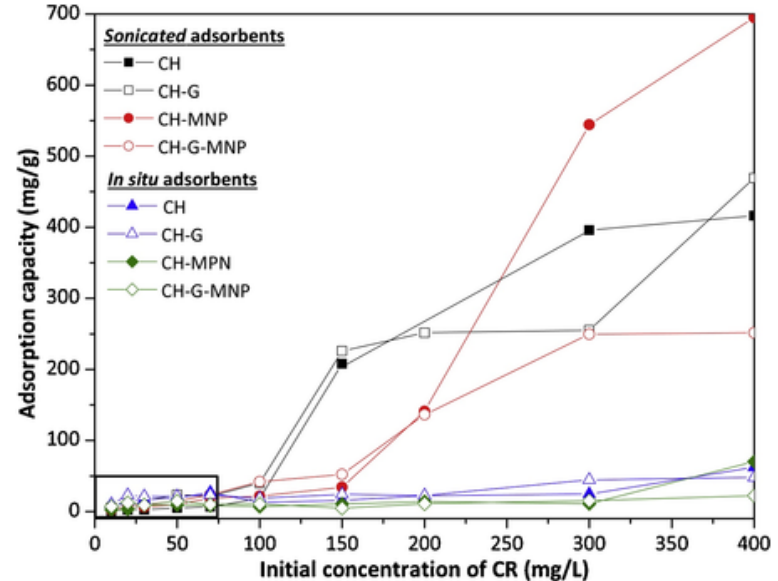

Fig. 1. Sorption capacity of chitosan based films as a function of the initial concentration of adsorbate (CR) solution.

Table 1

$\mathrm{pH}$ of $\mathrm{CR}$ solutions after and before adsorption of selected samples.

\begin{tabular}{|c|c|c|c|c|}
\hline Sample & & $\begin{array}{l}\text { CR initial } \\
\text { concentration }\left(\mathrm{mg} \mathrm{L}^{-1}\right)\end{array}$ & $\begin{array}{l}\mathrm{pH} \text { before } \\
\text { adsorption }\end{array}$ & $\begin{array}{l}\mathrm{pH} \text { after } \\
\text { adsorption }\end{array}$ \\
\hline \multirow[t]{8}{*}{ In situ } & $\mathrm{CH}$ & 40 & 7.90 & 7.50 \\
\hline & $\mathrm{CH}$ & 300 & 7.46 & 7.14 \\
\hline & $\mathrm{CH}-\mathrm{G}$ & 10 & 7.78 & 7.33 \\
\hline & $\mathrm{CH}-\mathrm{G}$ & 300 & 7.46 & 7.18 \\
\hline & $\begin{array}{l}\mathrm{CH}- \\
\mathrm{MNP}\end{array}$ & 30 & 7.52 & 7.56 \\
\hline & $\begin{array}{l}\mathrm{CH}- \\
\text { MNP }\end{array}$ & 150 & 7.30 & 7.48 \\
\hline & $\begin{array}{l}\text { CH-G- } \\
\text { MNP }\end{array}$ & 20 & 7.55 & 7.10 \\
\hline & $\begin{array}{l}\text { CH-G- } \\
\text { MNP }\end{array}$ & 200 & 7.42 & 7.16 \\
\hline \multirow[t]{8}{*}{ Sonicated } & $\mathrm{CH}$ & 20 & 7.55 & 4.71 \\
\hline & $\mathrm{CH}$ & 200 & 7.42 & 5.09 \\
\hline & $\mathrm{CH}-\mathrm{G}$ & 20 & 7.55 & 4.47 \\
\hline & $\mathrm{CH}-\mathrm{G}$ & 200 & 7.42 & 5.02 \\
\hline & $\begin{array}{l}\mathrm{CH}- \\
\text { MNP }\end{array}$ & 50 & 7.80 & 4.62 \\
\hline & $\begin{array}{l}\mathrm{CH}- \\
\mathrm{MNP}\end{array}$ & 300 & 7.63 & 5.07 \\
\hline & $\begin{array}{l}\text { CH-G- } \\
\text { MNP }\end{array}$ & 30 & 7.52 & 4.71 \\
\hline & $\begin{array}{l}\text { CH-G- } \\
\text { MNP }\end{array}$ & 150 & 7.30 & 4.61 \\
\hline
\end{tabular}

urally occurring linear polymer consists of two hydroxyl groups and one amino group in each repeating monomer, which gives it a high affinity for the adsorption of dyes and some metal ions that is enhanced by the presence of nitrogen heteroatoms (Massoudinejad,

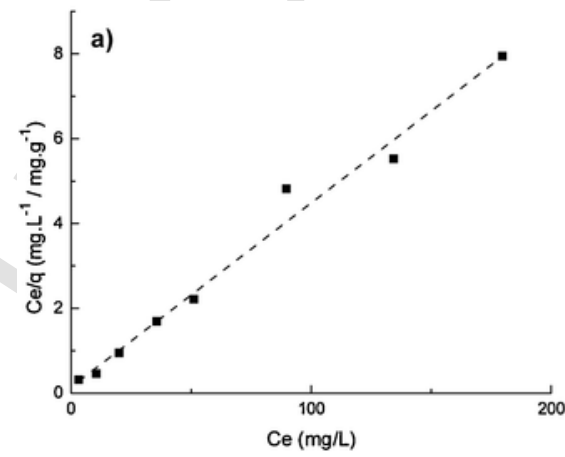

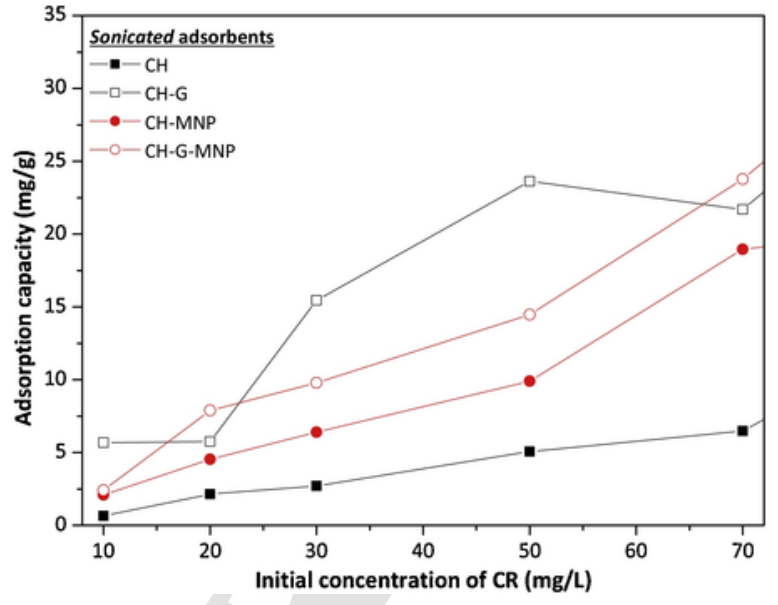

Fig. 2. Sorption capacity of sonicated films as a function of the initial concentration of adsorbate (CR) solution (low range).

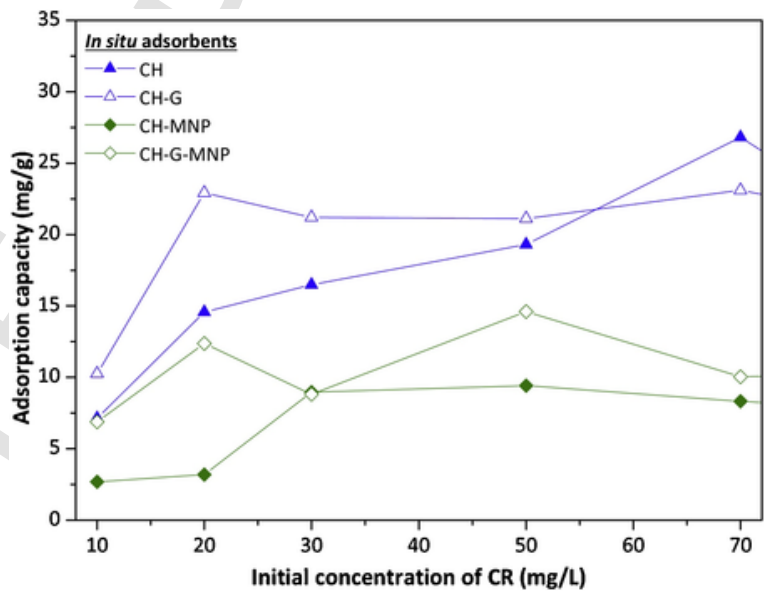

Fig. 3. Sorption capacity of in situ films as a function of the initial concentration of adsorbate (CR) solution (low range).

Rasoulzadeh, \& Ghaderpoori, 2019). Because of its good combination of properties, such as nontoxicity, low cost, biodegradability and plenty of hydroxyl, amino functional groups, etc., chitosan has been widely studied as potentially adsorbent for removal of dyes (Hui et al., 2018; Yang et al., 2018; You et al., 2018). Chitosan in its various forms i.e. flakes, beads, nanoparticles and powder has been reported for the treatment of polluted water and showed high adsorption efficiencies (Zahir et al., 2017). Recently, some chitosan-based adsorbents, characterized by fast adsorption kinetics, have been regarded as one of the most promising functional materials for environmental remediation

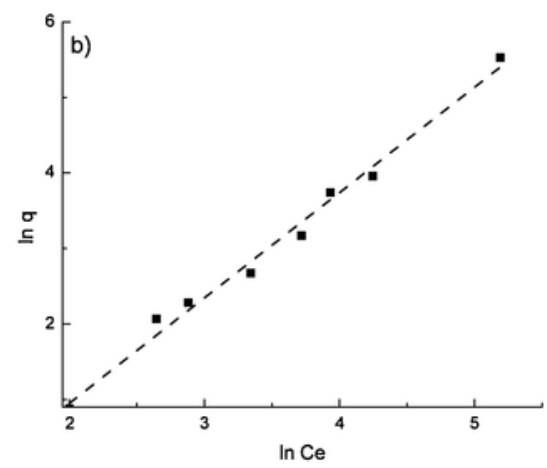

Fig. 4. Linearized plots of a) Langmuir isotherm applied to adsorption of CR onto CH-G in situ film; b) Freundlich isotherm applied to CH-G-MNP sonicated films. 
Table 2

Equilibrium isotherm parameters obtained for the different adsorbents.

\begin{tabular}{|c|c|c|c|c|c|c|c|}
\hline & $\mathrm{q}_{\max }\left(\mathrm{mg} \mathrm{L}^{-1}\right)$ & $\mathrm{K}_{\mathrm{L}}\left(\mathrm{Lmg}^{-1}\right)$ & $\mathrm{R}_{\mathrm{L}}\left(\mathrm{C}_{0}=10 \mathrm{mg} / \mathrm{L}\right)$ & $\mathrm{n}$ & $\mathrm{K}_{\mathrm{F}}$ & $\mathrm{R}^{2}$ & $\Delta \mathrm{G}^{0}(\mathrm{~kJ} / \mathrm{mol})$ \\
\hline \multicolumn{8}{|l|}{ In situ } \\
\hline $\mathrm{CH}$ & 19.34 & 0.13 & 0.43 & & & 0.859 & $-38,3$ \\
\hline CH-G & 23.07 & 0.30 & 0.25 & & & 0.981 & -40.3 \\
\hline CH-MNP & 12.86 & 0.03 & 0.75 & & & 0.910 & -34.8 \\
\hline CH-G-MNP & 10.30 & 1.89 & 0.05 & & & 0.990 & -44.9 \\
\hline \multicolumn{8}{|l|}{ Sonicated } \\
\hline $\mathrm{CH}$ & & & & 0.71 & 0.027 & 0.961 & \\
\hline CH-G & 16.95 & 0.06 & 0.61 & & & 0.866 & -36.4 \\
\hline CH-MNP & & & & 0.944 & 0.218 & 0.984 & \\
\hline CH-G-MNP & & & & 0.717 & 0.159 & 0.987 & \\
\hline
\end{tabular}
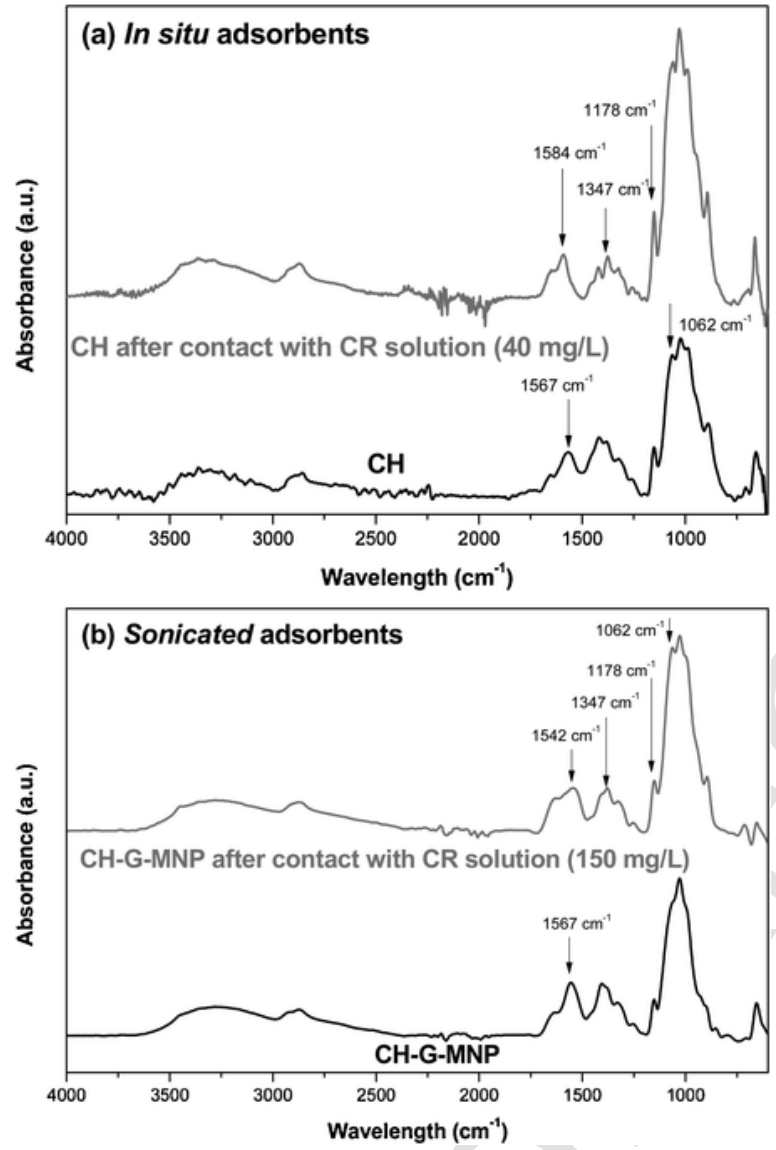

Fig. 5. FTIR spectrums of some of the films before and after CR adsorption: (a) films prepared by in situ method. (b) sonicated films.

(Hui et al., 2018; Pu, Ma, Zinchenko, \& Chu, 2017). In this line, magnetic chitosan adsorbents have been considered to be excellent candidates for heavy metals removal due to their biocompatibility (Zheng et al., 2019), highly chelating capability and easy magnetic separation (Li et al., 2017; Liu et al., 2018; Pu et al., 2017; Ren, Chen, Sun, Peng, \& Huang, 2014; Xu et al., 2015; You et al., 2018; Zheng et al., 2019). Compared to the long and tedious centrifugation separation process, the magnetic separation technology can be easily manipulated by an external magnet (You et al., 2018) and thus it has been considered as a promising environmental purification technique because it produces no contaminants during treatment and can remediate a large amount of wastewater within a short time (Ren et al., 2014; You et al., 2018). Es-
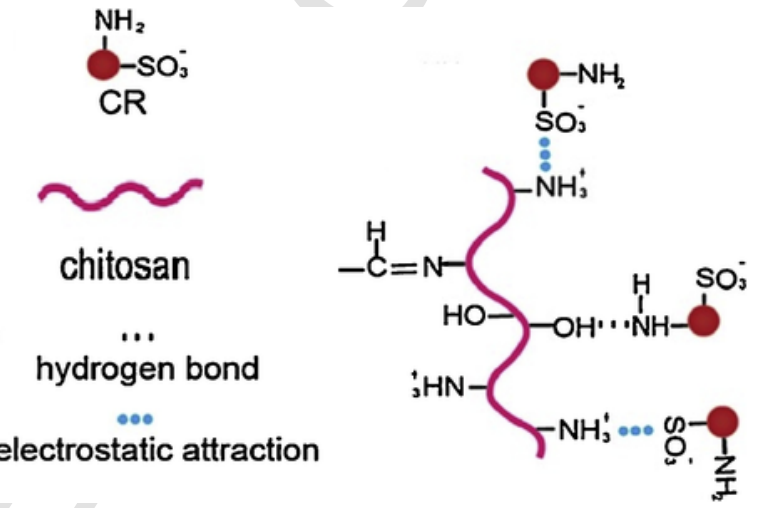

Fig. 6. Scheme for possible adsorption mechanism of congo red onto chitosan based fims. Adapted from Zheng et al. (2018). (For interpretation of the references to colour in this figure legend, the reader is referred to the web version of this article.)

pecially, many chitosan-based adsorbents are prepared with magnetic nanoparticles for their higher specific surface area and lower internal diffusion resistance (Galhoum et al., 2015; Kalkan, Aksoy, Aksoy, \& Hasirci, 2012; Li et al., 2017; Nasirimoghaddam, Zeinali, \& Sabbaghi, 2015; Thinh et al., 2013; Yuwei \& Jianlong, 2011). However, magnetic nanoparticles are highly chemically active, and are easily oxidized in the air which may result in magnetism loss and aggregation (Li et al., 2017; Reddy \& Lee, 2013). These nanoparticles also cause secondary pollution to the environment due to their very minute dimensions ( $\mathrm{Li}$ et al., 2017). On the other hand, we prepared and characterized thoroughly composite films made from magnetic iron oxides nanoparticles and chitosan by different, but relatively simple and cost-effective obtaining procedures (Kloster, Marcovich, \& Mosiewicki, 2015; Kloster, Muraca et al., 2015; Kloster et al., 2018). These films can perform as convenient and efficient natural polymer-based adsorbents, without the drawbacks associated to nano-particulate loose systems. In this line it should be noticed that loose particles are more difficult to manipulate in comparison with those contained into a macroscopically solid material like a film; their remotion after usage in water treatments is more expensive since, for instance, ultracentrifugation is often required when dealing with diamagnetic nano-particles; and, in addition, there are environmental issues related with nano-size materials handling, etc. Thus, in this paper, the anionic congo red (CR), a dye that can be metabolized to benzidine, a known human carcinogen (Hui et al., 2018), was selected as model pollutant to evaluate the adsorption behaviour of chitosan based magnetic composite films. and their respective matrices. Complementary techniques as SEM and FTIR studies, as well as isotherm modelling and thermodynamic analysis were used to interpret the adsorption results. 


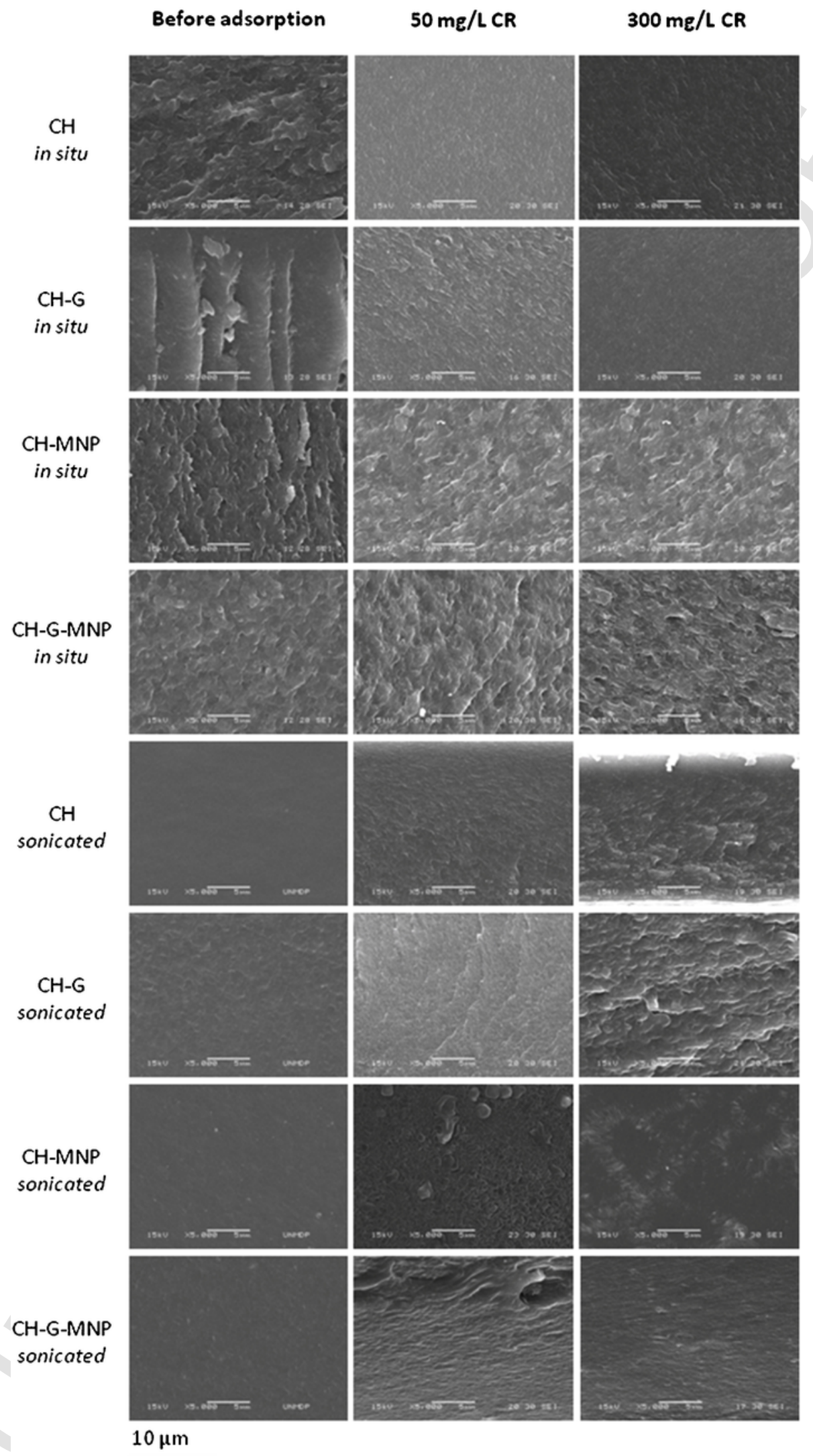

Fig. 7. SEM images obtained from the different adsorbent films: (a) before the adsorption, (b) after the adsorption of RC $50 \mathrm{mg} / \mathrm{L}$, (c) after the adsorption of RC $300 \mathrm{mg} / \mathrm{L}$. 
Table 3

Water content of in situ films after $24 \mathrm{~h}$ immersion in distilled water.

\begin{tabular}{ll}
\hline Sample & Absorbed water (wt.\%) \\
\hline CH & $72.7 \pm 1.6$ \\
CH-G & $81.4 \pm 3.1$ \\
CH-MNP & $58.7 \pm 3.9$ \\
CH-G-MNP & $60.2 \pm 5.3$ \\
\hline
\end{tabular}

\section{Experimental}

\subsection{Materials}

Congo red (CR) dye (Solarbio ${ }^{\circledR}$ ) of analytical grade without further purification was used as adsorbate. Congo red (CR, CI 22120, molecule weight $696.7 \mathrm{~g} \mathrm{~mol}^{-1}$ ) contains $\mathrm{NH}_{2}$ and $\mathrm{SO}_{3}{ }^{-}$functional groups and is an anionic diazo direct dye. Chitosan $(\mathrm{CH})$ (degree of deacetylation $98 \%, \mathrm{Mv}=1.61 \times 10^{5} \mathrm{~g} / \mathrm{mol}$ ), supplied by PARAFARM (Mar del Plata, Argentina) was used as received. Glycerol (G) purchased from SIGMA Aldrich was used as plasticizer. The ferric chloride $\left(\mathrm{FeCl}_{3}-6 \mathrm{H}_{2} \mathrm{O}\right)$, the ferrous sulfate $\left(\mathrm{FeSO}_{4}-7 \mathrm{H}_{2} \mathrm{O}\right)$, ammonium hydroxide and sodium hydroxide were obtained from Sigma-Aldrich.

\subsection{Methods}

\subsubsection{Preparation of the adsorbent materials}

The adsorbent materials based on chitosan $(\mathrm{CH})$ were synthesized following two different methods. In both methods the films were made with and without the addition of $30 \%$ wt. of glycerol (G) as plasticizer and with and without the addition of iron oxide nanoparticles (MNP). In situ films were prepared by a method where the iron oxide nanoparticles precipitated inside the solid polymer matrix. The detailed procedure for the preparation of these samples was presented in a previous publication (Kloster, Marcovich et al., 2015; Kloster, Muraca et al., 2015), however is also summarized here: chitosan solutions ( $2 \% \mathrm{wt} /$ v) were prepared in aqueous acetic acid $(1 \% \mathrm{v} / \mathrm{v})$, by magnetic stirring during $1.5 \mathrm{~h}$, adding the glycerol (if applicable, glycerol/chitosan wt. ratio $=0.3$ ) in the initial mixture. A $0.2 \mathrm{~mol} / \mathrm{L}$ iron salts solution was prepared by dispersing $9 \mathrm{~g}$ of ferric chloride with $4.62 \mathrm{~g}$ of ferrous sulfate $\left(\mathrm{Fe}^{2+}: \mathrm{Fe}^{3+}=1: 2 \mathrm{M}\right.$ ratio) in $250 \mathrm{~mL}$ of aqueous acetic acid $(1 \% \mathrm{v} / \mathrm{v})$. An appropriate volume of the iron salt solution (to obtain final films with $10 \mathrm{wt} \%$ nanomagnetite) was then dispersed into the $\mathrm{CH}$ solution, by magnetic stirring during $10 \mathrm{~min}$. The film-forming dispersions were defoamed under rest for one hour at room temperature, then poured into Teflon Petri dishes (diameter $=14 \mathrm{~cm}$ ), dried in a convective oven at $35^{\circ} \mathrm{C}$ for $24 \mathrm{~h}$, and kept under hood at room temperature for another day. Subsequently, the obtained films were peeled off from the plates, immersed in a $\mathrm{NaOH}$ aqueous solution $(5 \mathrm{~mol} / \mathrm{L})$ during $0.5 \mathrm{~h}$ to induce the chemical co-precipitation of $\mathrm{Fe}^{2+}$ and $\mathrm{Fe}^{3+}$ ions, and then washed several times with distilled water until neutralization. Finally, the films were dried again under hood at room temperature and then kept in a closed container containing dried silica gel at room temperature $\left(25 \pm 1^{\circ} \mathrm{C}\right)$ until testing. Conversely, sonicated nanocomposite films were made firstly synthesizing the MNP (Kloster et al., 2018). To obtain the MNP, $4.8 \mathrm{~g}$ of $\mathrm{FeSO}_{4} \cdot 7 \mathrm{H}_{2} \mathrm{O}$ and $9.32 \mathrm{~g}$ of $\mathrm{FeCl}_{3} \cdot 6 \mathrm{H}_{2} \mathrm{O}$ (molar ratio $\mathrm{Fe}^{+2}: \mathrm{Fe}^{+3}=1: 2$ ) were dissolved in approximately $40 \mathrm{~mL}$ of distilled water. Once the solution was perfectly homogenized, $15 \mathrm{~mL}$ of $\mathrm{NH}_{4} \mathrm{OH}$ was added drop by drop in order to avoid or minimize the MNPs aggregation. The suspension was stirred for $15 \mathrm{~min}$, allowing the complete precipitation of MNPs. After this time and with the help of a magnet placed out of the flask, several washes with distilled water were made until neutral $\mathrm{pH}$. Then, the wet particles were placed into a Petri plate and lyophilized to eliminate the water. Finally, the obtained powder was kept into a dark colored glass container. Then, the iron oxide nanoparticles were incorporated into the chitosan solution ( $2 \% \mathrm{w} / \mathrm{v}$ chitosan in $1 \% \mathrm{v} / \mathrm{v}$ aqueous acetic acid solution) at room temperature. Glycerol, in a weight ratio glycerol/chitosan equal 0.3 , was also added to the solution when applicable. $10 \mathrm{wt} \%$ MNPs were incorporated to the polymer solution to prepare nanocomposite films. Film forming suspensions were obtained by manually mixing MNPs with chitosan (or chitosan/glycerol) solution, followed by ultrasonication for $2 \mathrm{~h}$. Then, the suspensions were poured into Petri dishes (diameter $=14 \mathrm{~cm}$ ) and dried in a convective oven at $35^{\circ} \mathrm{C}$ for $24 \mathrm{~h}$, leading to solid films (Kloster et al., 2018). In both systems (in situ and sonicated samples), the MNPs present average diameter sizes around $10 \mathrm{~nm}$ (Kloster, Marcovich et al., 2015; Kloster et al., 2018).

The complete characterization of the films used in this work can be found in previous publications (Kloster, Marcovich et al., 2015; Kloster, Muraca et al., 2015; Kloster et al., 2018).

\subsubsection{Preparation of the adsorbate solution}

Congo red (CR) solutions with different concentrations (10-400 mg/ L) were prepared from a CR stock solution $(1000 \mathrm{mg} / \mathrm{L})$, by diluting the CR in bidestillated water. A calibration curve of CR was prepared by using a UV-vis spectrophotometer, measuring the absorbance of the different $\mathrm{CR}$ solutions at $\lambda=498 \mathrm{~nm}$, where the dye has a maximum absorbance when the $\mathrm{pH}$ of the solution is above 5 (Purkait, Maiti, Dasgupta, \& De, 2007; Tran, You, Hosseini-Bandegharaei, \& Chao, 2017). Therefore, for subsequent determinations, the concentration of CR was calculated using the linear regression equation of the calibration curve:

Absorbance $=0.041 \quad x$ Concentration of $\quad C R$ in $\mathrm{mg} / \mathrm{L}$

\subsubsection{Adsorption equilibrium studies}

The adsorption equilibrium studies were performed using a piece of film of $1 \times 1 \mathrm{~cm}^{2}$ as adsorbent. The adsorbent materials were previously dried in a vacuum oven at $60^{\circ} \mathrm{C}$ for $24 \mathrm{~h}$. Then, the samples were weighed and added to flasks with $25 \mathrm{~mL}$ of CR solution at a fixed concentration. The flasks were shaken $(60 \mathrm{rpm})$ for $23.5 \mathrm{~h}$ at room temperature $\left(25 \pm 1{ }^{\circ} \mathrm{C}\right)$. After this time, the film samples were allowed to stand for $0.5 \mathrm{~h}$ and then were extracted from the flasks using tweezers. Parts of the CR solutions used for the adsorption measurements were taken from the flasks and centrifuged for $5 \mathrm{~min}$ at $3500 \mathrm{rpm}$ (using a ROLCO centrifuge CM36R) and then their CR concentrations were assessed by UV-vis spectrophotometry. Two replicates of most of the samples were used in the adsorption tests. In those cases the average error in the measurements was below $10 \%$. The adsorption capacity of

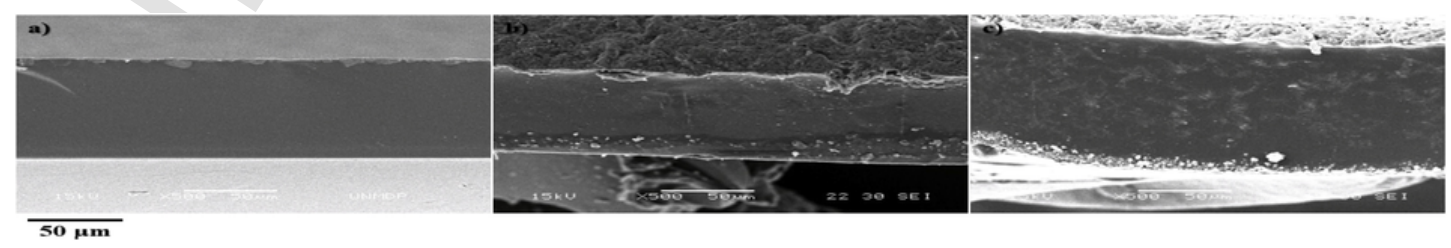

Fig. 8. SEM images obtained from the film CH-MNP sonicated: (a) before the adsorption, (b) after the adsorption of RC $50 \mathrm{mg} / \mathrm{L}$, (c) after the adsorption of RC $300 \mathrm{mg} / \mathrm{L}$. 
the films was calculated using the following equation:

$q=\frac{\left(C_{0}-C_{e}\right) \times V}{W}$

where $q$ represents the adsorption capacity ( $\mathrm{mg}$ of $\mathrm{CR} / \mathrm{g}$ adsorbent), $V$ is the volume of the solution and $W$ is the weight of adsorbent (film sample). The $\mathrm{pH}$ of the $\mathrm{CR}$ solution containing the adsorbent material was also measured in selected cases.

\subsubsection{Infrared spectroscopy (FTIR) characterization}

FTIR spectra of some of the films before and after CR adsorption were recorded by the attenuated total reflection technique (ATR) using a Perkin Elmer Spectrum 100 Fourier transform infrared spectrometer. Films were previously dried in a vacuum oven at $60^{\circ} \mathrm{C}$ for $24 \mathrm{~h}$. The spectra were recorded over a range of $500-4000 \mathrm{~cm}^{-1}$ with a resolution of $2 \mathrm{~cm}^{-1}$ and averaged over 32 scans.

\subsubsection{Scanning Electron Microscopy (SEM)}

The cross-section (obtained by cryo-fracture after immersing samples in liquid air) of the films before and after CR adsorption, were analyzed using a scanning electron microscope (JEOL, model JSM-6460 LV). For this purpose, the pieces of the films were mounted on bronze stubs using a double-sided tape and then coated with gold, before being observed under the microscope.

\section{Results and discussion}

\subsection{Adsorption equilibrium studies}

The adsorption curves for all the obtained films at different CR initial concentrations are shown in Fig. 1. Both kinds of films (in situ and sonicated) behave approximately in the same way in the zone of low initial concentrations. However, it is clear that there are two different adsorption zones for the sonicated films: up to $100 \mathrm{mg} / \mathrm{L}$ initial concentration, but when immersed in solutions of higher CR concentrations the sorption capacity of the sonicated films increases very much, reaching values of $700 \mathrm{mg} / \mathrm{g}$ in the best case. To understand this behavior, the $\mathrm{pH}$ of the $\mathrm{CR}$ solutions before and after adsorption was registered in selected cases and it is presented in Table 1. Clearly the $\mathrm{pH}$ of the adsorbent solution decreases after $24 \mathrm{~h}$ adsorption. However, in the case of in situ films the decrease is minimal and thus the solutions, independently of the CR concentration, remain always approximately neutral or slightly alkaline. It is worth to mention that the last step in the preparation of these in situ films involves the immersion of the film into an alkali solution $(\mathrm{NaOH}, 5 \mathrm{~mol} / \mathrm{L})$ during $0.5 \mathrm{~h}$ to induce the particle formation (chemical co-precipitation of $\mathrm{Fe}^{2+}$ and $\mathrm{Fe}^{3+}$ ions), and then successive washes until neutral $\mathrm{pH}$. Moreover, control films without nanoparticles were also prepared following this procedure for comparison. Accordingly, it is not surprising that the $\mathrm{pH}$ of the adsorbent solution changes very little after adsorption onto these neutral in situ films. On the contrary, the $\mathrm{pH}$ of the adsorbent solution becomes acidic after adsorption of sonicated samples, which again is in accordance with their preparation procedure (film forming dispersions obtained in aqueous acetic acid solution $(1 \% \mathrm{v} / \mathrm{v})$ by $2 \mathrm{~h}$ sonication, then casting and drying (Kloster et al., 2018)). Moreover, it was noticed that after sorption, in situ films remained visually entire, without cracks or fissures that lead us to think that part of the sample mass was lost during the test. Additionally no increase in the turbidity of the treated congo red solution was observed after adsorption tests involving in situ films. On the other hand and as was expected because chitosan is soluble in acidic pH (Lizardi-Mendoza, Monal, \& Valencia, 2016; Rinaudo, 2006), part of the sonicated samples were dissolved during the adsorption tests, which was also visualized as an increase in the treated congo red solution turbidity. In fact, before determining the final CR concentra- tion, the solutions had to be centrifugated to separate film pieces that could interfere with UV absorption measurements. Thus, the decrease of the $\mathrm{pH}$ in the case of sonicated samples could explain part of the observed behavior: firstly the partial dissolution of the films leads to samples with increased sorption area for the same sample mass, since now every small piece of the film contribute with its surface to the CR adsorption.

On the other hand, the $\mathrm{pH}$ of the dye solution is very important for the adsorption process, since it affects not only the surface charge of the adsorbent, but also the degree of ionization and the speciation of the adsorbate during the reaction (Thinh et al., 2013). Chatterjee, Chatterjee, Chatterjee, and Guha (2007) observed also that the adsorption of CR by chitosan beads had been found to be dependent on $\mathrm{pH}$ of the solution, i.e. it increased with the decrease in $\mathrm{pH}$. They also found that the surface charge of chitosan beads was positive in acidic $\mathrm{pH}$ and decreased gradually with increase in $\mathrm{pH}$, passing through zero potential at $\mathrm{pH}$ 6.4. In acidic $\mathrm{pH}$ range, surface charge of the adsorbent increases mainly due to increased protonation of amine group $\left(-\mathrm{NH}_{3}{ }^{+}\right)$of chitosan and since congo red is an acidic dye that contains negatively charged sulfonated group $\left(-\mathrm{SO}_{3}-\mathrm{Na}^{+}\right)$, they attributed the higher adsorption of the dye at low $\mathrm{pH}$ to the increase in electrostatic attraction between negatively charged dye molecule and positively charged amine group of chitosan. On the other hand, at $\mathrm{pH}$ higher than 6.4 where surface charge of chitosan samples is neutral, they indicated that adsorption of the dye may be attributed to physical forces only, which can explain the lower adsorption capacity of the samples made from the in situ methodology even when they were in contact with relatively concentrated CR solutions. Moreover, Zhu et al. (2012) also found that significant adsorption of the anionic dye onto chitosan coated magnetic iron oxide (adsorbent) still occurred at alkaline $\mathrm{pH}$ values, suggesting that the chemisorption mechanism might be operative. In addition, we confirmed in previous publications (Kloster, Marcovich et al., 2015; Kloster, Muraca et al., 2015) that in our in situ films strong interactions between the iron oxide particles and the amide/amino groups of the chitosan matrix were developed. In fact, Reddy and Lee (2013) indicated that one of the biggest advantages of preparing magnetic composites with chitosan is that it is quite easy to prepare chitosan-conjugated particles (chitosan-magnetite) using a co-precipitation method because the functional groups at the surface of iron oxide particles easily react with those of chitosan or its derivatives. Then, on the one hand, these interactions contribute to maintain the composite film integrity during the adsorption tests but for the other, they limit the adsorption capacity since some of the active sites should not be free to interact with the dye molecule.

Nevertheless, the adsorption behavior at lower CR concentrations deserves a more detailed analysis. Figs. 2 and 3 present an enlargement of the adsorption curves at low CR concentration for sonicated and in situ samples, respectively. It can be noted that there are both, similarities and differences between samples prepared from different methodologies. The first similarity is that the maximum adsorption capacity in the analyzed range is almost the same in both cases, which indicated that the $\mathrm{pH}$ is not the unique effect that should be taken into account. On the other hand, it is also observed that while the in situ films seem to reach saturation for CR concentrations of approximately $50 \mathrm{mg} / \mathrm{L}$, the sonicated samples continue to show a constant, although moderate, increase in CR adsorption when they are contacted with solutions of up to approximately $70 \mathrm{mg} / \mathrm{L}$ and then changes abruptly for higher concentrations, which could be indicating the existence of some critical concentration of CR that affects especially these films, probably accelerating the process of dissolution/fragmentation of the sample at concentrations greater than $70 \mathrm{mg} / \mathrm{L}$ and/or modifying its adsorption mechanism, something that clearly does not happen with in situ films.

Regarding the effect of the MNP on the CR adsorption capacity of the composites, their addition into the sonicated films leads to an in- 
crease for the non-plasticized composites, while the plasticized ones present a more random behavior. In the case of in situ films the adsorption capacity of the films is lower when they contain MNP in both cases (plasticized and not), which is directly related to the important interactions between the chitosan matrix and the MNP obtained by this preparation method that could "block" some interacting groups with the adsorbate, as indicated previously.

On the other hand, the presence of plasticizer seems to increase the adsorption capacity in both types of films. Glycerol increases the spacing between chitosan chains and, therefore, induces an increase in free volume, so that plasticized films would allow greater access of the contaminant to the adsorption sites of the polymer. At the same time, part of the glycerol is solubilized by contacting the films with water or aqueous solution, since the plasticizer is not chemically bonded to the bio-polymer, so the space occupied by the glycerol before coming into contact with water could be easily occupied by the adsorbate during the adsorption process. Anyhow, the expected plasticizer effect of glycerol increasing the access of CR is clearly observed at low initial concentrations of CR for the sonicated films (Fig. 2), while it is more subtle for the in situ samples (Fig. 3). This is again attributed to the differences in the preparation processes: the plasticized films made by the in situ methodology lose part of the added glycerol during the obtaining of the samples as will be further explained later and thus their actual glycerol content is lower than that of the sonicated films. On the other hand, at initial concentrations of dye higher than $70 \mathrm{mg} / \mathrm{L}$ (Fig. 1), the beneficial effect of the glycerol is not noticed either for the sonicated samples. In this case, probably the bigger CR molecule, added to its high concentration in the dye solution could exert a kind of "steric hindrance" effect, obstructing the diffusion of glycerol to the solution and thus, diminishing its positive effect in the adsorbent material.

Moreover, our CR adsorption results are well in the range of similar studies. For example, Zhu et al. (2012) found an adsorption capacity of CR (experimental results performed at $295 \mathrm{~K}$ ) of about $20 \mathrm{mg} / \mathrm{g}$ chitosan coated magnetic iron oxide particles when contacting the adsorbent particles ( $600 \mathrm{~min}$ contact time) with congo red solutions of initial concentrations up to $70 \mathrm{mg} / \mathrm{L}$ and Zheng et al. (2018) found that their dialdehyde microfibrillated cellulose/chitosan composite film is able to adsorb up to $40 \mathrm{mg} / \mathrm{g}$ CR when contacted with a $70 \mathrm{mg} / \mathrm{L}$ dye solution at $\mathrm{pH}=5.5$.

\subsection{Adsorption isotherms}

The adsorption isotherm is one of the most important considerations in designing adsorption systems, since it describes the interaction between an adsorbent and adsorbate. Thus, it is always considered as a major factor to determine the capacity of an adsorbent and optimization of the adsorbent consumption (Alimohammadi et al., 2017). Hence, the correlation of equilibrium data using either a theoretical or empirical equation is essential to interpret and predict the extent of adsorption (Gul et al., 2016). Langmuir isotherm, often applicable to a homogeneous adsorption surface with all the adsorption sites having identical adsorbate affinity, i.e. single-layer adsorption processes (Alimohammadi et al., 2017; Feng, Xiong, \& Zhang, 2015; Gul et al., 2016; Jeyaseelan, Chaudhary, \& Jugade, 2018), is represented as follows:

$q_{e}=\frac{K_{L} q_{\max } C_{e}}{1+K_{L} C_{e}}$

where $\mathrm{Ce}$ is the equilibrium concentration of the adsorbate $\left(\mathrm{mg} \mathrm{L}^{-1}\right)$, $\mathrm{q}_{\max }\left(\mathrm{mg} \mathrm{g}^{-1}\right)$ and $\mathrm{K}_{\mathrm{L}}\left(\mathrm{Lmg}^{-1}\right)$ are the Langmuir constants related to the maximum adsorption capacity and the energy of adsorption, respectively. $\mathrm{q}_{\max }$ and $\mathrm{b}$ are calculated from the slopes and intercepts of the straight lines of plot of $1 /$ qe $v$ s. $1 /$ Ce. The favorability of the adsorption process in the Langmuir model can be determined by means of the
$R_{L}$ dimensionless factor $\left(R_{L}=1 /\left(1+K_{L} C_{0}\right)\right.$, with $R_{L}=0,0<R_{L}<1$, $\mathrm{R}_{\mathrm{L}}=1$, and $\mathrm{R}_{\mathrm{L}}>1$ indicating irreversible, favorable, linear, and unfavorable adsorption isotherms, respectively (Alimohammadi et al., 2017; Jeyaseelan et al., 2018).

Freundlich isotherm, based on adsorption on multi-layer, nonuniform (Alimohammadi et al., 2017; Massoudinejad et al., 2019) and heterogeneous surface (Alimohammadi et al., 2017; Gul et al., 2016), is commonly represented as;

$q_{e}=K_{F} C_{e}^{\frac{1}{n}}$

$\mathrm{K}_{\mathrm{F}}\left(\mathrm{mg}^{1-1 / \mathrm{n}} \mathrm{L}^{1 / \mathrm{n}} \mathrm{g}^{-1}\right)$ and $\mathrm{n}$ (dimensionless) are the Freundlich constants, characteristics of the system showing adsorption capacity and adsorption intensity (or heterogeneity factor), respectively (Alimohammadi et al., 2017; Feng et al., 2015; Gul et al., 2016; Jeyaseelan et al., 2018). Values of $\mathrm{n}$ between 1 and 10 indicate favorable adsorption (Jeyaseelan et al., 2018), while $1 / \mathrm{n}$ represents the exponent of nonlinearity (i.e., C-type, L-type, and S-type isotherms).

Linear diagrams of Langmuir and Freundlich adsorption isotherms applied to selected samples are shown in Fig. 4. Table 2 presents the equilibrium isotherm parameters obtained for the different adsorbents. The constants of the Langmuir and/or Freundlich models were only calculated when the $\mathrm{R}^{2}$ (regression coefficient) resulted higher than 0.85. Moreover, other two-parameter models like Temkin and Dubinin-Radushkevich ones (Alimohammadi et al., 2017) were also applied to our experimental data, but they showed an even poorer fit.

From the results presented in Table 2 it is clear that all in situ samples and only one of the sonicated specimens ( $\mathrm{CH}-\mathrm{G})$ respond to Langmuir model, while the others are best fitted by the Freundlich isotherm. According to Langmuir isotherm model, a monolayer coverage of dyes over surface films is expected (Gul et al., 2016). Moreover, it is clear that specimens without MNP present higher values of maximum adsorption/uptake capacity $\left(\mathrm{q}_{\max }\right.$ ) of $\mathrm{CR}$ than their counterparts with magnetic particles, although it is not possible their removal using a magnet. As expected, this reasoning confirms the previous statements regarding the blocking of some active adsorption sites of the chitosan matrix by the interaction developed with the MNP. Furthermore, in all cases where the best fitting was obtained with the Langmuir isotherm, the $\mathrm{R}_{\mathrm{L}}$ values, calculated using the lower initial concentration, are between 0 and 1 , which not only strongly supports the favorability of the adsorption reaction (Massoudinejad et al., 2019), but also confirms that electrostatic interactions between films samples and CR were developed. Regarding experimental data that follow Freundlich model, it is clear that most of the sonicated films respond to a multilayer and uneven adsorption of the adsorbate on a heterogeneous surface with lack of uniform distribution of energy and with the interaction between adsorbed molecules (Jeyaseelan et al., 2018; Massoudinejad et al., 2019), which again is consistent with the fragmentation of these films during adsorption tests. Table 2 shows that the $\mathrm{n}$ values are lower than 1 , and thus indicative of S-type isotherms (1/n values are higher than 1$)$. According to Alimohammadi et al. (2017) this behavior can be ascribed to that some compounds containing a polar functional group may be in competition with water for adsorption sites at low concentration ranges. On the other hand, $\mathrm{K}_{\mathrm{F}}$ values, which are related with the adsorption capacity of the adsorbent (Jeyaseelan et al., 2018), are higher for the samples containing MNP, in contrast to what was observed for the in situ adsorbents.

For those adsorbents film samples that follows the Langmuir isotherm, the standard free energy change $\left(\Delta G^{0}\right)$ was calculated as $\Delta G^{0}=-R T \ln K$ (Feng et al., 2015; Jeyaseelan et al., 2018; Kumar et al., 2009), being $\mathrm{R}$ the gas constant $\left(8.314 \mathrm{~J} \mathrm{~mol}^{-1} \mathrm{~K}^{-1}\right)$, $\mathrm{T}$ the absolute temperature ( $298 \mathrm{~K}$ in this case) and $\mathrm{K}$ the equilibrium constant related to the Langmuir constant $\mathrm{K}_{\mathrm{L}}$ (with units of $\mathrm{L} \mathrm{mol}^{-1}$ ) as $\mathrm{K}=\mathrm{K}_{\mathrm{L}} * 55.5$, where the value 55.5 corresponds to the molar concentration of the 
solvent (in this case water) with units of $\mathrm{molL}^{-1}$ (Kumar et al., 2009). These values are included in Table 2 and are comparable to that reported by Kumar et al. (2009) for fluoride adsorption onto granular ferric hydroxide, while are one order of magnitude higher than those reported by Feng et al. (2015) for adsorption of congo red on cross-linked porous chitosan films and Gul et al. (2016) for adsorption of cationic or anionic dyes onto $\mathrm{Fe}_{3} \mathrm{O}_{4}$ supported chitosan-graphene oxide composite nano-particles. As expected, all calculated $\Delta G^{0}$ are negative, indicating spontaneous nature of the adsorption processes involving in situ and CH-G sonicated adsorbent samples.

\subsection{FTIR and SEM studies}

To better understand the adsorption results, FTIR spectra of the films were obtained before and after CR adsorption. Fig. 5a shows the spectra of $\mathrm{CH}$ prepared by in situ methodology and Fig. 5b those of the CH-G-MNP samples prepared by sonication procedure, being the observed differences applicable, respectively, to the other samples obtained with the same preparation method. As can be seen, the presence of CR generates a change in the intensity of some peaks, especially in the bands near 1062 and $1178 \mathrm{~cm}^{-1}$, which could be associated with the stretching $\mathrm{S}=\mathrm{O}$ in the dye and in the band at $1347 \mathrm{~cm}^{-1}$, associated to the presence of the aromatic amine of the dye (Acemioğlu, 2004; Silverstein, Webster, \& Kiemle, 2005). Notice that for neat chitosan a band at $1065 \mathrm{~cm}^{-1}$, attributed to the combined effects of $\mathrm{C}-\mathrm{N}$ stretching vibration of primary amines and the $\mathrm{C}-\mathrm{O}$ stretching vibration from the primary alcohol, added to a band at $1380 \mathrm{~cm}^{-1}$, coming from the primary amine of chitosan are expected (Feng et al., 2015; Liu, Chen, Huang, \& Lai, 2016). Thus, the effect of the adsorbed CR is just to increase the intensity of these peaks. Moreover, in the spectrum of the in situ $\mathrm{CH}$ film, the band appearing at $1567 \mathrm{~cm}^{-1}$ (Kloster, Marcovich et al., 2015), assigned to the $\delta s \mathrm{NH}^{+}$bending mode of the ammonium side chains of the biopolymer, shifts to $1584 \mathrm{~cm}^{-1}$ after CR adsorption; in the case of the sonicated CH-G-MNP sample, it shifts from $1557 \mathrm{~cm}^{-1}$ (before adsorption) to $1542 \mathrm{~cm}^{-1}$ (after adsorption). These shifts were also taken as proofs of the interaction between adsorbent and adsorbate in other related papers (Janaki et al., 2012). This confirms that at least the $\mathrm{N}-$ $\mathrm{H}$ bonds of the $\mathrm{N}$-acetyl group (amide II) took part in the adsorption reaction. Thus, according to the previous findings, a scheme of the adsorption mechanism is presented in Fig. 6. It is inferred that the adsorption driving forces may include two parts: electrostatic attraction as well as the hydrogen bonding. The electrostatic attraction is due to the protonated amino groups of chitosan chains and the sulfonate groups of the dye and it is the main mechanism for adsorption at acidic $\mathrm{pH}$ (Chatterjee et al., 2007; Zheng et al., 2018). In the FTIR spectra it appears as a shift in the peak at $1567 \mathrm{~cm}^{-1}$. However, at higher pHs where surface charge of chitosan based adsorbents is neutral, adsorption of the dye may be attributed to physical forces (i.e. hydrogen bonds, van der Waals forces, etc.) only. According to Chatterjee et al. (2007), there is every possibility of hydrogen bond and electrostatic forces formation between some of the molecular components of congo red such as $\mathrm{N}, \mathrm{S}, \mathrm{O}$, benzene ring and $\mathrm{CH}_{2} \mathrm{OH}$ groups of the chitosan molecule. According to Janaki et al. (2012) these interactions should be observed as a significant shift in the wavenumber $3421 \mathrm{~cm}^{-1}$ (neat chitosan based adsorbent) to $3333 \mathrm{~cm}^{-1}$ for $\mathrm{CR}$ adsorption. However, this is a quite complex IR region since the contributions of $\mathrm{O}-\mathrm{H}$ stretching and amino groups are present (Feng et al., 2015) and thus, in the present case no clear shift could be observed.

The microstructure of the films was analyzed once the adsorption was finished, through SEM microscopy. Fig. 7 shows the fracture surface (thickness) of the films tested (5000 $\times$ magnification). For the in situ nanocomposites, both plasticized and unplasticized, no significant changes were observed after adsorption. As indicated in a previous publication (Kloster, Marcovich et al., 2015), the corresponding com- posites absorb less moisture than their respective matrices and thus, it is certainly expected that they suffer less changes in their microstructure due to swelling during the adsorption tests. Again this is attributed to the very strong interactions between MNP and chitosan matrix that probably limit the penetration of the dye solution into the film, and, therefore, the changes suffered after absorption are only observed on the film surfaces, that become red due to the strong coloration of the adsorbed dye. In addition, the plasticized films made by the in situ methodology lose part of the added glycerol during the obtaining of the samples (i.e. the chemical precipitation of the magnetic particles using $\mathrm{NaOH}$ as the last step of composite film preparation that involves immersion of the film in $\mathrm{NaOH}$ solution and then several washing steps with distilled water until neutralization) and they can lose even more during the immersion of the sample in the aqueous solution of CR. Thus, even when the actual glycerol content of the in situ films submitted to adsorption test is unknown, we believe it is only slightly larger than that of the samples containing no glycerol. To confirm this reasoning, the amount of liquid water absorbed by the in situ films was determined and it is presented in Table 3. It should be noticed that the role of glycerol, as plasticizer, is to interact with the polymer chains and change their three-dimensional organization, decreasing the attractive intermolecular forces and increasing the free volume and chain mobility of the polymeric material (Zheng et al., 2018). Actually, in plasticized polymers the polymer-polymer physical cross-links (hydrogen bonds and/or hydrophobic interactions) are replaced by polymer-plasticizer interactions (Pommet, Redl, Guilbert, \& Morel, 2005; Zubeldía, Ansorena, \& Marcovich, 2015), which should enhance their hydrophilic nature (Kloster et al., 2018). However, as it can be noticed from Table 3, the water absorbed by the in situ samples seems to be more dependent of the MNP content than on the nominal glycerol content ( $30 \mathrm{wt} . \%$ of the total sample mass), confirming that part of the plasticizer was lost during film preparation.

At the same time, the sonicated films exhibit so large swelling when immersed in water that their equilibrium water content could not be quantified (Kloster, 2019). Accordingly, the microstructure of these samples changes so much after adsorption tests since not only part of the glycerol is lost into the CR aqueous solution but also due to the great swelling of the films upon contact with dye solution. This behavior explains to a certain extent the greater adsorption capacity of these films. In addition, the MNP are less anchored to the matrix, which allows the polymer chains and/or nanoparticles to attain greater mobility when in contact with water (co-plasticizer), resulting in greater changes in the microstructure of the samples after adsorption. Moreover, in Fig. 8, which shows the images of the sonicated sample-CH-MNP with lower magnification $(500 \times)$, it can be corroborated that after $24 \mathrm{~h}$ of contact with the CR solution the structure of the composite film changes from smooth and uniform to presenting numerous irregularities, changes that seem to be independent of the CR concentration. This could be because the material lost part of its free chitosan, while the MNP surrounded by the bio-polymer remain in the films.

\section{Conclusions}

Chitosan based composite films with different glycerol and iron oxide contents, prepared by two different procedures were tested as potential adsorbents films for congo red. As a general trend it was found that the equilibrium adsorption capacity increases with the increased initial dye concentration and decreases with the increased $\mathrm{pH}$, which is related to the driving force of the concentration gradient and electrostatic interactions, respectively. However, these changes are not very prominent when the adsorbents are contacted with dye solutions containing less than $70 \mathrm{mg} / \mathrm{L}$ congo red. The $\mathrm{pH}$ of the congo red solutions was slightly alkaline at the beginning of the tests and then decreased just a little when contacting with in situ films or a lot when a sonicated 
film is tested as adsorbent. In addition, the strong interactions developed among iron oxide particles and chitosan matrix in the in situ films limited the amount of groups available for dye adsorption and thus, the sorption capacity of these nanocomposite samples is lower than that those of the corresponding matrices and also lower than those of the sonicated ones. On the other hand, the in situ films remained intact during the adsorption time while the sonicated ones dissolved partially leading to an adsorbent material formed by many smaller parts and consequently, increased sorption area. On the other hand, the presence of plasticizer seems to increase the adsorption capacity in both types of films by allowing greater access of the contaminant to the adsorption sites of the polymer.

\section{Acknowledgements}

The authors gratefully acknowledge the financial support provided by the Science and Technology National Promotion Agency (ANPCyT, Grant PICT-2016-2034) and the National University of Mar del Plata (Project \# 15/G494 - ING500/17).

\section{References}

Acemioğlu, B., 2004. Adsorption of congo red from aqueous solution onto calcium-rich fly ash. Journal of Colloid and Interface Science 274 (2), 371-379.

Alimohammadi, M., Saeedi, Z., Akbarpour, B., Rasoulzadeh, H., Yetilmezsoy, K., Al-Ghouti, M.A., Khraisheh, M., McKay, G., 2017. Adsorptive removal of arsenic and mercury from aqueous solutions by eucalyptus leaves. Water, Air, \& Soil Pollution 228 (11), 429.

Chatterjee, S., Chatterjee, S., Chatterjee, B.P., Guha, A.K., 2007. Adsorptive removal of congo red, a carcinogenic textile dye by chitosan hydrobeads: Binding mechanism, equilibrium and kinetics. Colloids and Surfaces A: Physicochemical and Engineering Aspects 299 (1-3), 146-152.

Feng, T., Xiong, S., Zhang, F., 2015. Application of cross-linked porous chitosan films for congo red adsorption from aqueous solution. Desalination and Water Treatment 53 (7), 1970-1976

Galhoum, A.A., Mafhouz, M.G., Abdel-Rehem, S.T., Gomaa, N.A., Atia, A.A., Vincent, T., et al., 2015. Cysteine-functionalized chitosan magnetic nano-based particles for the recovery of light and heavy rare earth metals: Uptake kinetics and sorption isotherms. Nanomaterials 5 (1), 154-179.

Gul, K., Sohni, S., Waqar, M., Ahmad, F., Norulaini, N.N., AK, M.O., 2016. Functionalization of magnetic chitosan with graphene oxide for removal of cationic and anionic dyes from aqueous solution. Carbohydrate Polymers 152, 520-531.

Hui, M., Shengyan, P., Yaqi, H., Rongxin, Z., Anatoly, Z., Wei, C., 2018. A highly efficient magnetic chitosan "fluid" adsorbent with a high capacity and fast adsorption kinetics for dyeing wastewater purification. Chemical Engineering Journal 345, 556-565.

Janaki, V., Oh, B.-T., Shanthi, K., Lee, K.-J., Ramasamy, A., Kamala-Kannan, S., 2012. Polyaniline/chitosan composite: An eco-friendly polymer for enhanced removal of dyes from aqueous solution. Synthetic Metals 162 (11-12), 974-980.

Jeyaseelan, C., Chaudhary, N., Jugade, R., 2018. Sulphate-crosslinked chitosan as an adsorbent for the removal of congo red dye from aqueous solution. Air, Soil and Water Research 11, 1178622118811680.

Kalkan, N.A., Aksoy, S., Aksoy, E.A., Hasirci, N., 2012. Adsorption of reactive yellow 145 onto chitosan coated magnetite nanoparticles. Journal of Applied Polymer Science 124 (1), 576-584.

Kloster, G.A., 2019. Síntesis y caracterización de películas magnéticas de matriz biopolimérica con propiedades adsorbentes (Synthesis and characterization of magnetic films of biopolymer matrix with adsorbent properties. $\mathrm{PhD}$ in Material Science, National University of Mar del Plata.

Kloster, G.A., Marcovich, N.E., Mosiewicki, M.A., 2015. Composite films based on chitosan and nanomagnetite. European Polymer Journal 66, 386-396.

Kloster, G.A., Muraca, D., Meiorin, C., Pirota, K.R., Marcovich, N.E., Mosiewicki, M.A., 2015. Magnetic characterization of chitosan-magnetite nanocomposite films. European Polymer Journal 72, 202-211.

Kloster, G.A., Muraca, D., Londoño, O.M., Knobel, M., Marcovich, N.E., Mosiewicki, M.A., 2018. Structural analysis of magnetic nanocomposites based on chitosan. Polymer Testing 72, 202-213.

Kumar, E., Bhatnagar, A., Ji, M., Jung, W., Lee, S.-H., Kim, S.-J., Lee, G., Song, H., Choi, J.-Y., Yang, J.-S., 2009. Defluoridation from aqueous solutions by granular ferric hydroxide (GFH). Water Research 43 (2), 490-498.

Li, J., Jiang, B., Liu, Y., Qiu, C., Hu, J., Qian, G., Guo, W., Ngo, H.H., 2017. Preparation and adsorption properties of magnetic chitosan composite adsorbent for $\mathrm{Cu} 2+$ removal. Journal of Cleaner Production 158, 51-58.
Liu, K., Chen, L., Huang, L., Lai, Y., 2016. Evaluation of ethylenediamine-modified nanofibrillated cellulose/chitosan composites on adsorption of cationic and anionic dyes from aqueous solution. Carbohydrate Polymers 151, 1115-1119.

Liu, B., Chen, X., Zheng, H., Wang, Y., Sun, Y., Zhao, C., et al., 2018. Rapid and efficient removal of heavy metal and cationic dye by carboxylate-rich magnetic chitosan flocculants: Role of ionic groups. Carbohydrate Polymers 181, 327-336.

Lizardi-Mendoza, J., Monal, W.M.A., Valencia, F.M.G., 2016. Chemical characteristics and functional properties of chitosan. Chitosan in the preservation of agricultural commodities. Elsevier, 3-31.

Massoudinejad, M., Rasoulzadeh, H., Ghaderpoori, M., 2019. Magnetic chitosan nanocomposite: Fabrication, properties, and optimization for adsorptive removal of crystal violet from aqueous solutions. Carbohydrate Polymers 206, 844-853.

Nasirimoghaddam, S., Zeinali, S., Sabbaghi, S., 2015. Chitosan coated magnetic nanoparticles as nano-adsorbent for efficient removal of mercury contents from industrial aqueous and oily samples. Journal of Industrial and Engineering Chemistry 27, 79-87.

Pommet, M., Redl, A., Guilbert, S., Morel, M.-H., 2005. Intrinsic influence of various plasticizers on functional properties and reactivity of wheat gluten thermoplastic materials. Journal of Cereal Science 42 (1), 81-91.

$\mathrm{Pu}$, S., Ma, H., Zinchenko, A., Chu, W., 2017. Novel highly porous magnetic hydrogel beads composed of chitosan and sodium citrate: An effective adsorbent for the removal of heavy metals from aqueous solutions. Environmental Science and Pollution Research 24 (19), 16520-16530.

Purkait, M.K., Maiti, A., Dasgupta, S., De, S., 2007. Removal of congo red using activated carbon and its regeneration. Journal of Hazardous Materials 145 (1-2), 287-295.

Reddy, D.H.K., Lee, S.-M., 2013. Application of magnetic chitosan composites for the removal of toxic metal and dyes from aqueous solutions. Advances in Colloid and Interface Science 201, 68-93.

Ren, Y., Chen, Y., Sun, M., Peng, H., Huang, K., 2014. Rapid and efficient removal of cationic dyes by magnetic chitosan adsorbent modified with EDTA. Separation Science and Technology 49 (13), 2049-2059.

Rinaudo, M., 2006. Chitin and chitosan: Properties and applications. Progress in Polymer Science 31 (7), 603-632.

Silverstein, R.M., Webster, F.X., Kiemle, D.J., 2005. Spectrometric identification of organic compounds. John Wiley \& Sons.

Thakur, S., Pandey, S., Arotiba, O.A., 2016. Development of a sodium alginate-based or ganic/inorganic superabsorbent composite hydrogel for adsorption of methylene blue. Carbohydrate Polymers 153, 34-46.

Thakur, S., Pandey, S., Arotiba, O.A., 2017. Sol-gel derived xanthan gum/silica nanocomposite-A highly efficient cationic dyes adsorbent in aqueous system. Internationa Journal of Biological Macromolecules 103, 596-604.

Thinh, N.N., Hanh, P.T.B., Hoang, T.V., Hoang, V.D., Dang, L.H., Van Khoi, N., et al., 2013 Magnetic chitosan nanoparticles for removal of $\mathrm{Cr}$ (VI) from aqueous solution. Materials Science and Engineering C 33 (3), 1214-1218.

Tran, H.N., You, S.-J., Hosseini-Bandegharaei, A., Chao, H.-P., 2017. Mistakes and inconsistencies regarding adsorption of contaminants from aqueous solutions: A critical review. Water Research 120, 88-116.

Xu, Y., Dang, Q., Liu, C., Yan, J., Fan, B., Cai, J., et al., 2015. Preparation and characterization of carboxyl-functionalized chitosan magnetic microspheres and submicrospheres for $\mathrm{Pb} 2+$ removal. Colloids and Surfaces A: Physicochemical and Engineering Aspects $482,353-364$.

Yang, X., Li, Y., Gao, H., Wang, C., Zhang, X., Zhou, H., 2018. One-step fabrication of chitosan-Fe $(\mathrm{OH}) 3$ beads for efficient adsorption of anionic dyes. International Journal of Biological Macromolecules 117, 30-41.

You, L., Huang, C., Lu, F., Wang, A., Liu, X., Zhang, Q., 2018. Facile synthesis of high performance porous magnetic chitosan-polyethylenimine polymer composite for congo red removal. International Journal of Biological Macromolecules 107, 1620-1628.

Yuwei, C., Jianlong, W., 2011. Preparation and characterization of magnetic chitosan nanoparticles and its application for Cu (II) removal. Chemical Engineering Journal 168 (1), 286-292.

Zahir, A., Aslam, Z., Kamal, M.S., Ahmad, W., Abbas, A., Shawabkeh, R.A., 2017. Development of novel cross-linked chitosan for the removal of anionic congo red dye. Journal of Molecular Liquids 244, 211-218.

Zheng, X., Li, X., Li, J., Wang, L., Jin, W., Pei, Y., et al., 2018. Efficient removal of anionic dye (congo red) by dialdehyde microfibrillated cellulose/chitosan composite film with significantly improved stability in dye solution. International Journal of Biological Macromolecules 107, 283-289.

Zheng, C., Zheng, H., Wang, Y., Sun, Y., An, Y., Liu, H., et al., 2019. Modified magnetic chitosan microparticles as novel superior adsorbents with huge "force field" for capturing food dyes. Journal of Hazardous Materials 367, 492-503.

Zhu, H., Zhang, M., Liu, Y., Zhang, L., Han, R., 2012. Study of congo red adsorption onto chitosan coated magnetic iron oxide in batch mode. Desalination and Water Treatment 37 (1-3), 46-54.

Zubeldía, F., Ansorena, M.R., Marcovich, N.E., 2015. Wheat gluten films obtained by compression molding. Polymer Testing 43, 68-77. 